\title{
ESTRUCTURAS DE FÁBRICA DE LADRILLO Y BLOQUE CERÁMICO NO ARMADA
}

\author{
(UNREINFORCED LOADBEARING WALLS MADE OF CERAMIC \\ BRICKS AND BLOCKS) \\ F. de Isidro Gordejuela \\ Colegio Universitario CEU- Arquitectura. Madrid
}

ESPAÑA

Fecha de recepción: 2-VI-95

$631-14$

\section{RESUMEN}

En esta exposición se presenta un procedimiento de cálculo para muros de fábrica de ladrillo y bloque cerámico. Dicho procedimiento está basado en el método de comprobación de las tensiones admisibles en las secciones críticas al igual que la normativa vigente en nuestro pais ${ }^{\prime}$, y sigue la estructura del futuro Eurocódigo EC.6.3.

El método considera la colaboración conjunta de los muros ante acciones horizontales yverticales teniendo en cuenta los distintos modos de comportamiento estructural de cada fábrica. Se contempla la reducción de la capacidad resistente de la fábrica debida a los factores de esbeltez y excentricidad, considerando el efecto de los empotramientos muro-forjado, cambios de sección entre plantas, acciones horizontales, apilastramientos, arriostramientos y tolerancias de ejecución.

Con carácter previo se hacen una serie de consideraciones sobre las características mecánicas de los materiales cerámicos, ladrillos y bloques.
SUMMARY

We present a method for the design of brick and ceramic blocks loadbearing walls. This methods is based on the verification of design stresses in critical points of the wall structure.

The method considers the interaction and the connection among loadbearing and shear walls designed to carry imposed loads and lateral forces, taking into account the structural behaviour of each wall. Stength reduction factors due to slenderness and eccentricity, bending moments caused by floor loads, wall columns, stiffening walls, accidental eccentricities or the effect of lateral forces are analysed.

The basic mechanical properties of ceramic materials, compressive and shear strength are previous defined.

\section{Generalidades}

Los edificios construidos con muros portantes de ladrillo o bloque cerámico son concebidos como un sistema tridimensional formado por forjados y muros sensiblemente perpendiculares entre sí. La estructura debe tener forma celular, debiendo prevalecer esta idea siempre frente a la de elementos de carga paralelos.

La transmisión de las cargas hasta la cimentación se realiza a través de muros de carga (en el caso de cargas verticales gravitatorias) y de muros de arriostramiento (que ante acciones horizontales se comportan como ménsulas de gran canto).

Los muros de carga se encuentran normalmente sometidos a flexocompresión. Los empotramientos de los forjados en los muros, los cambios de sección de los muros de fachada en plantas bajas, el viento contra sus caras o las tolerancias de ejecución producen excentricidades de carga en el plano perpendicular al muro, cuya inflüencia en la resistencia del muro debe estimarse. 
Los muros de arriostramiento, sometidos simultáneamente a acciones horizontales y cargas verticales, se encuentran sometidos a flexocompresión y corte. En este caso debería considerarse la aparición de flexiones que provocan también excentricidades de carga en el propio plano del muro.

\section{Hipótesis de cálculo}

En la estructura deben comprobarse al menos estas dos hipótesis generales:

\section{A. CARGA + SOBRECARGA \\ B. CARGA + VIENTO}

A. Carga permanente + sobrecarga: Esta hipótesis tiene por objeto comprobar la resistencia a compresión de los muros de carga en las secciones consideradas, como efecto más desfavorable.

B. Carga permanente + viento: Esta hipótesis verifica la resistencia a compresión y a corte de los muros de arriostramiento, no incluyendo sobrecargas.

Debe aclararse que un muro cargado siempre puede tener un efecto arriostrante en la estructura; en ese sentido, muchos muros de estructuras de fábrica son comprobados simultáneamente como muros de carga en la hipótesis A, y como muros de arriostramiento en la hipótesis B.

El proyectista establece cuál es el comportamiento de cada muro planta por planta, con el fin de delimitar razonablemente qué secciones críticas deben comprobarse.

\section{Hipótesis generales}

Se establecen cuatro hipótesis generales de funcionamiento en las estructuras de muros de fábrica:

\section{- la fábrica no admite tracciones}

- se acepta que las resultantes de las cargas verticales pasan por el eje en la parte inferior del muro.

- a efectos de cálculo en muros con huecos, sólo se considera como muro resistente al machón que queda entre los bordes de éstos.

- la elevada rigidez de los planos de forjado permite suponer que las cargas se distribuyen entre los muros según la capacidad de cada uno para resistirlos, es decir, proporcionalmente a su rigidez.

\section{Secuencia de Cálculo}

El método que se expone en este documento no es propiamente de dimensionamiento de secciones, sino de comprobación; es decir, debe hacerse una estimación o realizarse un precálculo para establecer una sección de fábrica que posteriormente se comprueba.

Parafacilitar la realización del método general debe seguirse paso a paso la secuencia de cálculo siguiente:

a) Determinar los siguientes parámetros en los distintos tipos de muros en el edificio:

- resistencia característica a compresión, $\mathrm{f}_{\mathrm{k}}$,

- resistencia característica al corte, $\mathrm{f}_{\mathrm{vk}}$

- coeficiente de seguridad $\gamma$, a emplear por el proyectista,

- tensión admisible a compresión $\sigma_{a}$

- tensión admisible al corte $\tau_{\mathrm{a}}$

- espesor virtual $t_{v}$, y altura virtual $h_{v}$

b) Analizar planta por planta cada uno de los elementos resistentes (o al menos los más desfavorables). Determinar cuál es su comportamiento.

c) Tener en cuenta las cargas acumuladas en plantas superiores, con la reducción de sobrecargas que proceda ${ }^{5}$. Contabilizar cargas y acciones en cada muro (al menos en los más desfavorables).

d) Estudiar en cada muro las excentricidades de carga y deducir las de cálculo en la parte superior $e_{1}$, y en la central $\mathrm{e}_{2}$. Determinar el espesor virtual $\mathrm{t}_{\mathrm{v}} \mathrm{y}$ el coeficiente de excentricidad $\mathrm{m}=6 \mathrm{e} / \mathrm{t}_{\mathrm{v}}$

e) Determinar la altura virtual $h_{v}$ del muro analizado, en función de sus vínculos laterales. Determinar la esbeltez virtual de cada muro $\Gamma$, a partir de conocer su altura virtual $h_{v}$ y su espesor virtual $t_{v}$.

f) Obtener el coeficiente $\Phi$, de reducción de la resistencia por esbeltez y excentricidad de cargas.

g) Por último, obtener la tensión a la que está sometida la fábrica y compararla con la admisible.

Si el valor de la tensión en la fábrica no supera la tensión admisible, el dimensionamiento del muro es válido, prosiguiéndose con el cálculo. Si por el contrario, es mayor, hay que reconsiderar esta comprobación, variando las características del muro incrementando su resistencia (la del ladrillo o la del mortero), aumentando el espesor, el número de vínculos o reduciendo el tamaño de los huecos.

La determinación de los parámetros que aparecen en la secuencia de cálculo es sencilla y se explica a continuación.

\subsection{Determinación de parámetros relativos al material}

a) Resistencia característica a compresiō $f_{k}$, y resistencia característica al corte $f_{v k}$ 
La resistencia característica a compresión $\mathrm{f}_{\mathrm{k}}$ del material que compone el muro se define como la resistencia por debajo de la cual, no considerando el efecto de la excentricidad, esbeltez u otra restricción, puede encontrarse solamente el $5 \%$ de los resultados de todas las medidas de resistencia efectuadas. De modo equivalente se define la resistencia característica al corte $\mathrm{f}_{\mathrm{vk}}$, aquella por debajo de la cual pueden solamente encontrarse el $5 \%$ de los resultados de todas las medidas de resistencia efectuadas.

En la práctica, ambas tensiones se estiman a partir de la resistencia de ladrillos y morteros. Existen numerosas fórmulas empíricas o tablas en las distintas normativas existentes que permiten la obtención de estos parámetros ${ }^{6}$. En ese sentido es recomendable considerar los valores que propone la norma española para las fábricas de ladrillo, que a su vez están en función de otros parámetros, como la plasticidad del mortero o el espesor de la junta ${ }^{6}$.

La resistencia al corte del muro $f_{v k}$, definida como la resistencia al efecto combinado de fuerzas horizontales y cargas verticales en el plano del muro, viene dada por la relación:

$$
\mathrm{f}_{\mathrm{vk}}=\mathrm{f}_{\mathrm{vk} 0}+0,4 \sigma
$$

siendof $_{\mathrm{vk} 0}$ la resistencia al corte ${ }^{7}$ para $\sigma=0$

y $\sigma$ la tensión de cálculo a compresión vertical del muro considerado que coexiste con la tensión tangencial, en la condición más desfavorable.

\section{b) Coeficiente de seguridad}

La seguridad se introduce a través de un coeficiente global que minora la resistencia de las fábricas y que tiene en cuenta también una mayoración de las cargas. Se tomará como valor para el coeficiente global de seguridad $\gamma=5$.

c) Tensión admisible a compresión $\sigma_{\mathrm{a}}$, y tensión admisible al corte $\tau_{\mathrm{a}}$

Las tensiones admisibles o de comparación resultan de dividir el valor de las resistencias características por el coeficiente de seguridad global $\gamma$.

\subsection{Parámetros relativos a la geometría de los muros}

a) Espesor virtual, $t_{v}$

El espesor virtual de un muro de sección resistente no rectangular sería igual al espesor de un muro de sección rectangular que tuviera un comportamiento resistente equivalente al primero. El empleo de este parámetro simplifica la comprobación de muros y considera posibles apilastramientos o machones de formas complejas ${ }^{8}$. b) Altura virtual, $h_{v}$

Laaltura virtual se obtiene a partir de la siguiente expresión:

$$
\mathrm{h}_{\mathrm{v}}=\alpha \cdot \mathrm{h}
$$

donde $\alpha$ es un factor vínculo lateral ${ }^{9}$

Este factor es menor a medida que el número de arriostramientos laterales es mayor, o que la distancia entre muros laterales de arriostramiento disminuye. Se considera que un muro siempre está arriostrado en cabeza y pie por los forjados.

\subsection{Comportamiento del muro. Excentricidades de carga}

Un muro de fábrica está sometido generalmente a cargas verticales, acciones horizontales de viento y a momentos producidos por los empotramientos muro-forjado o debidos a ensanchamientos del muro portante inferior en un nudo.

Cualquier combinación de cargas y momentos puede sustituirse por una carga que afecte al muro con una determinada excentricidad. La metodología que se propone trabaja siempre con excentricidades, haciendo el proceso de cálculo más cómodo y sencillo (Fig. 1).
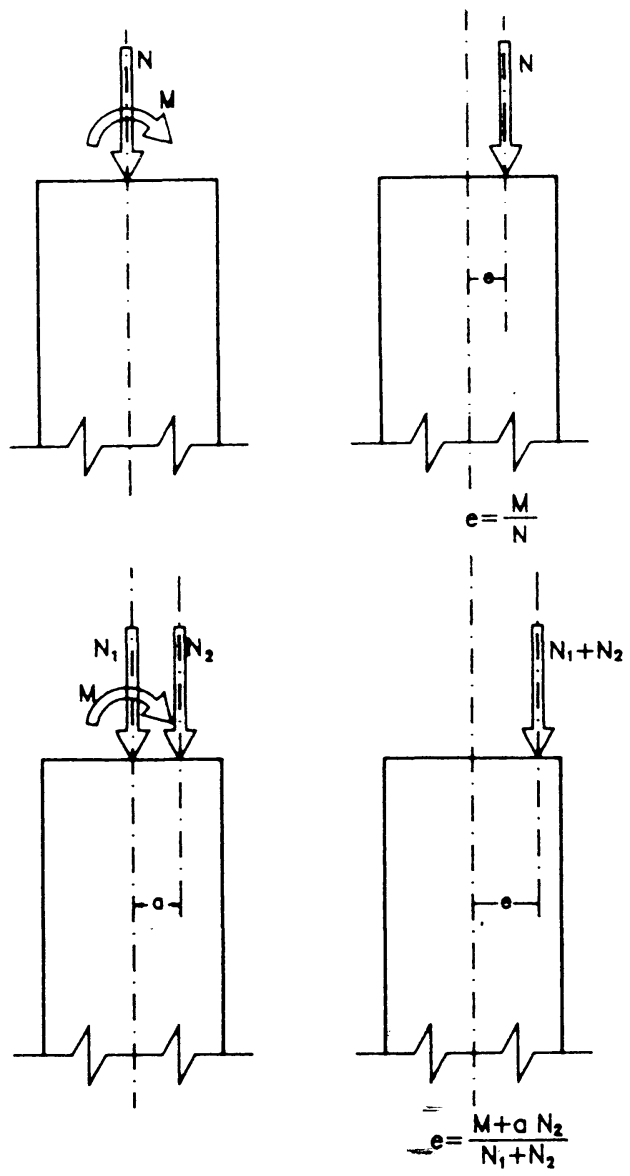

Fig. 1 
La excentricidad de cálculo resulta de la combinación más desfavorable de algunos de los siguientes términos:

excentricidad $\mathrm{e}_{\mathrm{w}}$, debida a acciones horizontales sobre el muro expuesto,

excentricidad $\mathrm{e}_{\mathrm{F}}$, debida al empotramiento producido entre el forjado y la fábrica,

excentricidad $e_{R}$, debida al posible ensanchamiento del muro portante inferior en un nudo,

excentricidad $\mathrm{e}_{\mathrm{A}}$, debida a tolerancias de ejecución

\section{a) Excentricidad debida al viento.}

En este caso deben comprobarse los muros de arriostramiento, cuya excentricidad $e_{\mathrm{wb}}$ se mide en el plano del muro, y los muros de carga y cerramiento, que reciben la carga de viento perpendicularmente a su plano, cuya excentricidad $\mathrm{e}_{w_{t}}$ se mide en dirección perpendicular al plano del muro. En los muros que actúan como muros de carga y de arriostramiento coexisten ambas excentricidades.

El momento que puede tomarse para el cálculo de $\mathrm{e}_{\mathrm{w}_{\mathrm{t}}}$ es el isostático, suponiendo el muro biapoyado en su pie y su coronación. Esta hipótesis estaría del lado de la seguridad, puesto que la rigidez de la unión muro-forjado no es despreciable.

b) Excentricidad debida al empotramiento en la unión muro-forjado.

El momento flector que se produce en el muro en su unión con el forjado podría ser determinado por un cálculo más o menos exacto, teniendo en cuenta las rigideces correspondientes y las posibles tracciones que se producen en la fábrica cuando la resultante de fuerzas está aplicada fuera del núcleo central de inercia. Sin embargo la hipótesis de empotramiento perfecto entre muros y forjados es discutible en muchos casos; la fábrica cerámica es un material que apenas resiste tracciones y su capacidad para trabajar a flexión es limitada ${ }^{10}$.

Pueden adoptarse como valores de momentos en los nudos los recogidos en la tabla 1 .

TABLA 1

Valor de los momentos en la unión muro-forjado.

\begin{tabular}{|ccc|}
\hline Caso & $\begin{array}{c}\text { Número de forjados encima } \\
\text { del nudo considerado } \\
1 \text { ó más }\end{array}$ \\
\hline $\mathrm{A}$ & 0 & $\mathrm{p} \cdot \mathrm{I}^{2} / 100$ \\
\hline $\mathrm{B}$ & 0 & $\mathrm{p} \cdot \mathrm{I}_{1}^{2} / 160$ \\
\hline $\mathrm{C}$ & 0 & $\mathrm{D} / 400$ \\
\hline
\end{tabular}

donde $D=p \cdot I_{M}^{2}-g \cdot I_{N}^{2} \quad\left(I_{M}>I_{N}\right)$

siendo

p carga total por unidad de longitud;

g carga permanente por unidad de longitud;

$I_{M}$ luz mayor;

Iv luz menor.
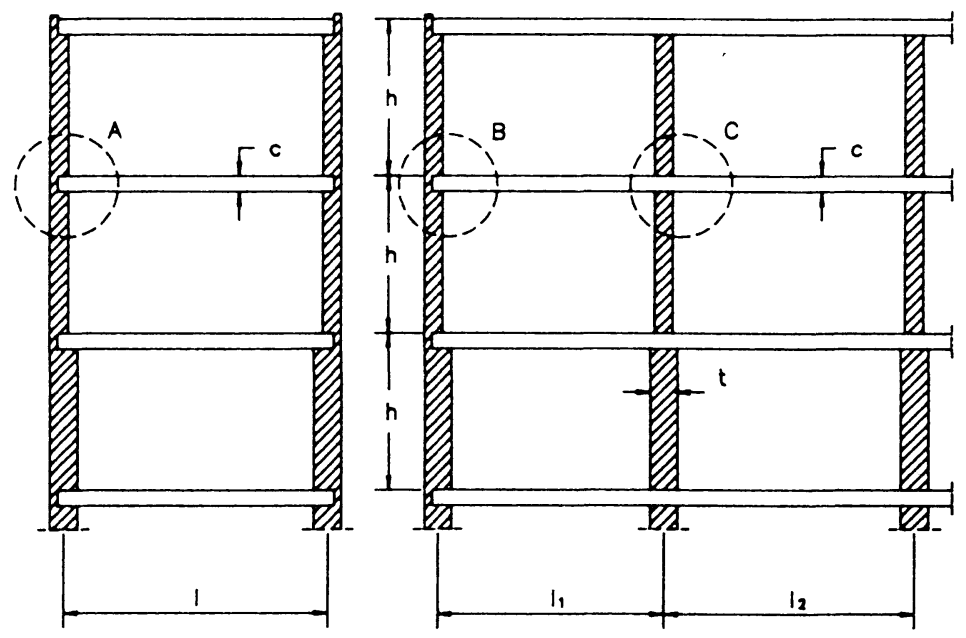

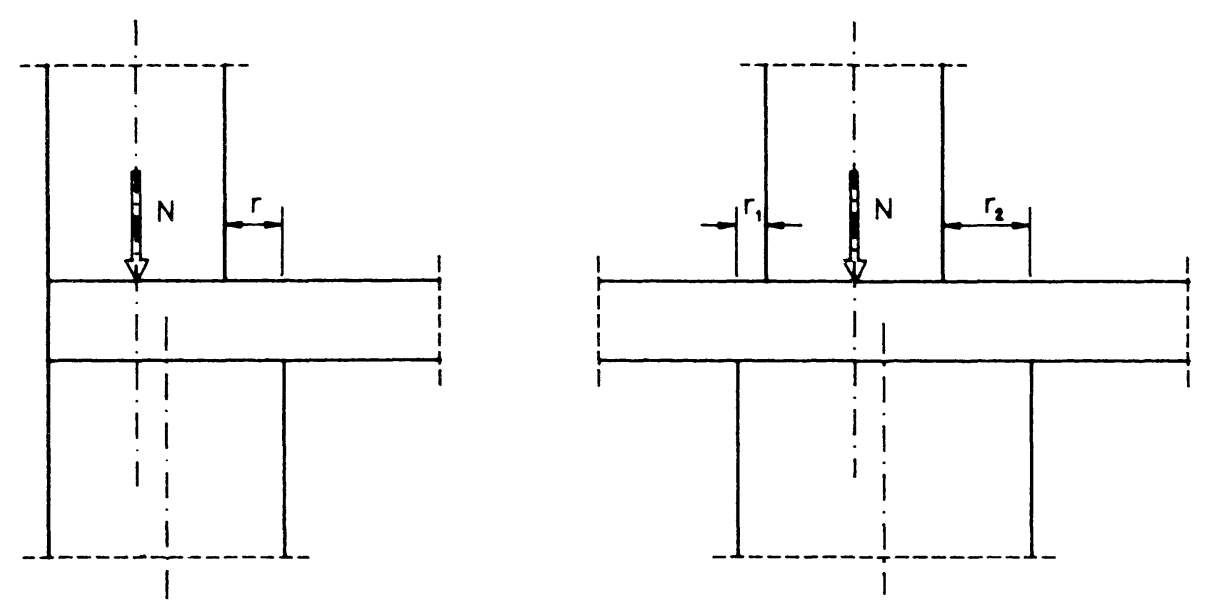

Fig. 2

La excentricidad $e_{\mathrm{F}}$ se calcula a partir de estos valores de momentos divididos por la carga total debida al forjado en el nudo.

c) Excentricidad debida al aumento de espesor de un muro.

En la hipótesis de que la carga del muro superior se considera centrada, los valores que toma $\mathrm{e}_{\mathrm{R}}$ son los que muestra la figura $2^{11}$.

d) Excentricidad debida a tolerancias de ejecución o accidental.

La excentricidad accidental considera las tolerancias derivadas de la técnica de ejecución de los muros de piezas cerámicas. La excentricidad $\mathrm{e}_{\mathrm{A}}$ debe tomar el valor:

$$
e_{A}=h / 300
$$

donde $h$ es la altura del muro entre caras superiores de forjados, expresada en $\mathrm{cm}$.

\subsection{Determinación de la excentricidad para el cálculo}

a) Determinación de la excentricidad en muros de carga.

Debido a que los valores de las distintas excentricidades no son continuos a lo largo del muro, $y$ teniendo en cuenta las hipótesis simplificativas que se adoptan en el funcionamiento de la fábrica, parece conveniente tomar un valor de cálculo para la excentricidad que esté del lado de la seguridad, en toda la altura del muro.

Las distintas excentricidades recogidas a lo largo del apartado anterior pueden ser de dos tipos diferentes:

-Excentricidades en cabeza de muro, como son $\mathrm{e}_{\mathrm{F}} \mathrm{y} \mathrm{e}_{\mathrm{R}}$. $\mathrm{Su}$ valor varía linealmente a lo largo del muro, desde un máximo en el extremo superior hasta cero.
-Excentricidad debida a cargas horizontales $e_{w_{t}}$, normalmente de viento. Su valor varía según una parábola, de manera análoga al diagrama de momentos flectores en una pieza biapoyada. Toma un valor máximo en el centro del muro y un valor de excentricidad cero en la cabeza y el pie del muro. En la figura 3 se indica la forma en que se combinan los dos tipos de excentricidades.

El valor de excentricidad que debe utilizarse para el cálculo resulta de la más desfavorable de estas dos combinaciones:

$$
\begin{aligned}
& e_{1}=\left|e_{F}+e_{R}\right|+\left|e_{A}\right| \leq 0,33 \\
& e_{2}=e_{1} / 2+\left|e_{w_{t}}\right| \leq 0,33
\end{aligned}
$$

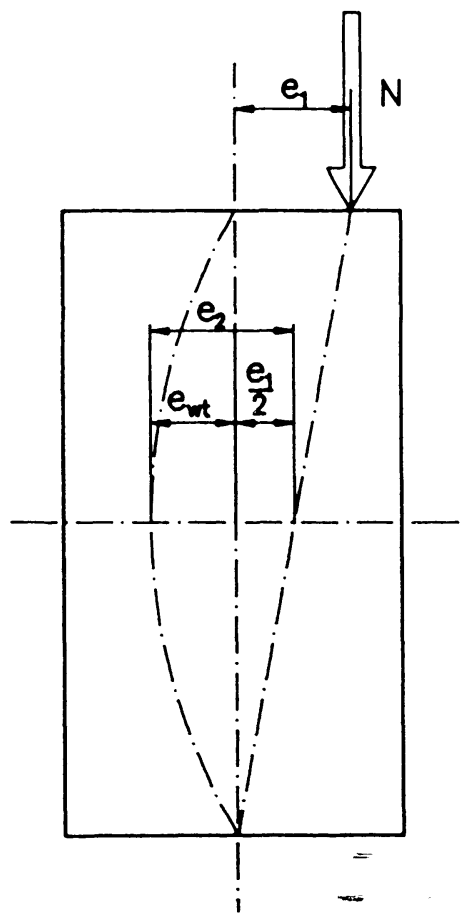

Fig. 3 
$e_{1}$ comprueba el valor máximo de la excentricidad en la cabeza del muro

$\mathrm{e}_{2}$ comprueba el valor máximo de la excentricidad en la sección donde el momento debido al viento es mayor

Para el cálculo se toma la más desfavorable de las dos, y se contabiliza perpendicularmente al plano del muro.

b) Determinación de la excentricidad en los muros de arriostramiento

La combinación del momento flector en cualquier sección del muro de arriostramiento debido a la acción de las fuerzas horizontales $\mathrm{M}$ y la resultante de las fuerzas verticales $\mathrm{N}$ puede ser sustituida por la resultante $\mathrm{N}$ con una excentricidad $\mathrm{e}_{\mathrm{w} \text {. }}$. Esta excentricidad se contabiliza en el propio plano del muro.

\subsection{Coeficiente de excentricidad y esbeltez virtual}

El coeficiente de excentricidad de cargas relaciona los valores de excentricidad y espesor del muro. Se define por la siguiente expresión:

$$
\mathrm{m}=6 \cdot \mathrm{e} / \mathrm{t}_{\mathrm{v}}
$$

siendo e el valor de la excentricidad de cálculo y $t_{v}$ el espesor virtual del muro

El valor del coefíciente de excentricidad indica el estado de tensiones de una sección de muro y entre otros puede tomar los siguientes valores (Fig. 4):

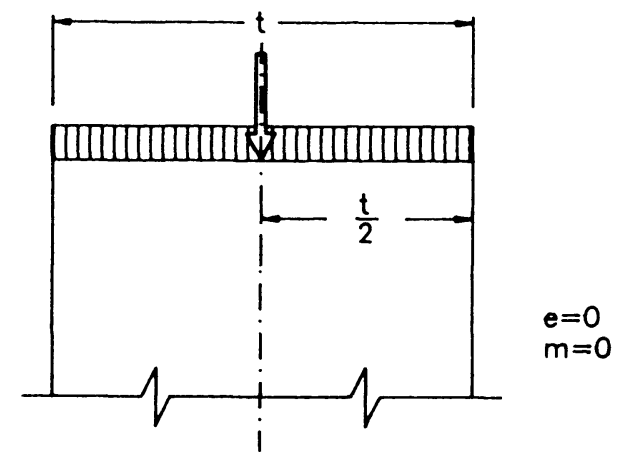

- cuando $m<1$, la resultante cae dentro del tercio medio de la sección, es decir, la ley de tensiones es trapezoidal y la sección está comprimida.

- cuando $m=1$, el diagrama de tensiones es triangular y toda la sección se encuentra comprimida, siendo la tensión nula en una de las caras del muro.

- cuando $m>1$, el diagrama es triangular, con base igual a tres veces la distancia de la resultante al borde de la sección más próximo. Debido a esto, aparecen partes de la sección traccionadas que no se consideran resistentes a efectos de su colaboración a resistir momentos.

Es deseable que no se produzcan tracciones o que estas estén controladas. Normalmente no deben admitirse valores superiores $a_{t}=2,00$ en muros cargados verticalmente (que equivale a media sección traccionada), y de $\mathrm{m}_{\mathrm{b}}=1,30$ en muros de arriostramiento ${ }^{12}$, siendo $\mathrm{m}_{\mathrm{t}}$ la excentricidad medida perpendicularmente al plano del muro, $\mathrm{y}_{\mathrm{b}}$ la excentricidad medida en el propio plano del muro.

4.6.Comprobación a flexocompresión. Obtención del coeficiente de reducción de resistencia por esbeltez y excentricidad de cargas

a) Muros de carga

El coeficiente penaliza la resistencia del muro de fábrica debido al efecto de la esbeltez y la excentricidad de la carga. Dicho coeficiente se obtiene en función del coeficiente de excentricidad $m_{t}$ y de la esbeltez y su valor se encuentra tabulado $^{13}$ (ver tabla 2).

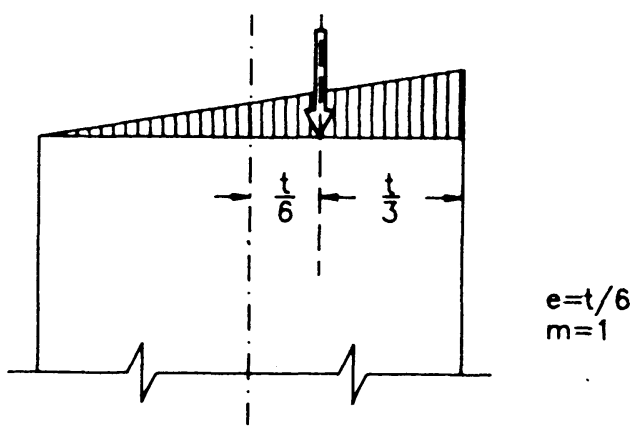

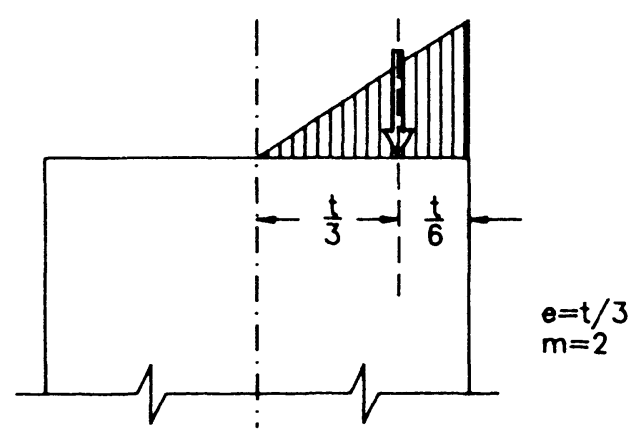

Fig. 4 
Valores del coeficiente $\Phi$.

\begin{tabular}{|rrrrrrr|}
\hline$\alpha \frac{h}{t}$ & & \multicolumn{5}{c|}{ Coef. de excentricidad $m=6$ eAt } \\
\cline { 4 - 8 } & 0,00 & 0,50 & 1,00 & 1,50 & 2,00 \\
\hline$<5$ & 1,00 & 0,76 & 0,63 & 0,46 & 0,33 \\
\hline 5 & 0,96 & 0,72 & 0,56 & 0,39 & 0,27 \\
\hline 10 & 0,86 & 0,62 & 0,45 & 0,27 & 0,15 \\
\hline 15 & 0,70 & 0,49 & 0,32 & 0,17 & - \\
\hline 20 & 0,56 & 0,37 & 0,23 & - & - \\
\hline 25 & 0,46 & 0,28 & - & - & - \\
\hline
\end{tabular}

$\varnothing$

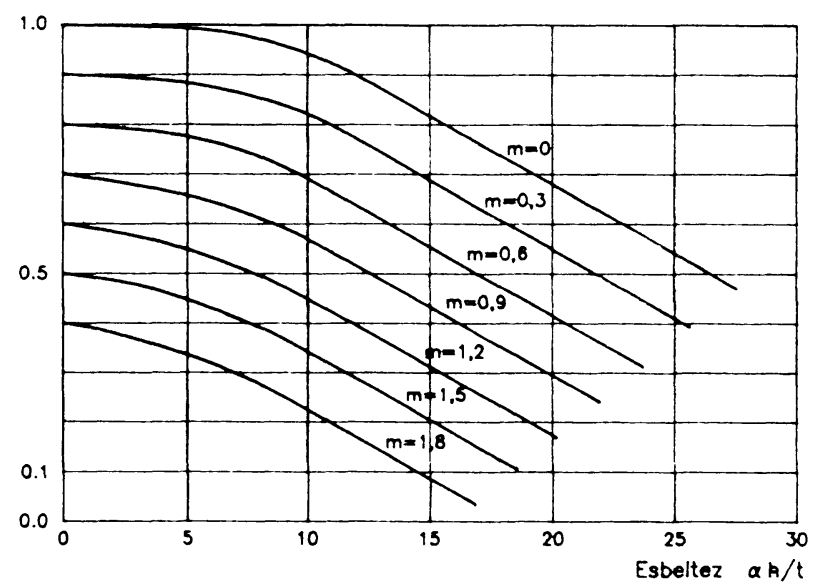

Para valores no incluidos en la tabla se admite la interpolación lineal. En ningún caso se admiten extrapolaciones.

Para comprobar la sección de un muro sobre el que actúa una carga $N$, la condición de seguridad que debe cumplirse es la siguiente:

$$
\sigma=\frac{N}{\Phi A} \leq \sigma_{a} \quad(A=t \cdot b)
$$

siendo:
$\sigma \quad$ la tensión de cálculo a compresión vertical
$\sigma_{\mathrm{a}}$ la tensión admisible a compresión
$\mathrm{N}$ la carga vertical en la base del muro
$\Phi$ el coeficiente de reducción por esbeltez y excentricidad
A el área neta de la sección horizontal de muro
$t$ espesor del muro
b longitud del muro

b) Muros de arriostramiento

En un muro de arriostramiento cargado debe tenerse en cuenta que coexisten dos excentricidades de carga: una perpendicular al planodel muro $m_{t}$ debida a excentricidades de carga vertical, y otra en el propio plano del muro $\mathrm{m}_{\mathrm{b}}$ debida a acciones horizontales absorbidas por los forjados.

Consideraremos, por tanto, dos coeficientes de reducción por esbeltez y excentricidad:

- $\Phi_{t}$ coefíciente de reducción de la resistencia del muro en función de la esbeltez y la excentricidad transversal $m_{t}$

- $\Phi_{b}$ coeficiente de reducción de la resistencia del muro en función de la esbeltez y la excentricidad longitudinal $\mathrm{m}_{\mathrm{b}}$. Este coeficiente se obtiene considerando el valor de la esbeltez $<5$, ya que en esta dirección el espesor del muro a considerar coincide con su longitud, valor que es apreciablemente grande respecto a su altura en la mayoría de los casos.

La condición de seguridad que debe cumplirse es la siguiente:

$$
\sigma=\frac{N}{\Phi_{t} \cdot \Phi_{b} \cdot A}
$$

\subsection{Comprobación al corte.}

Para comprobar la sección de un muro sobre el que actúa la acción horizontal de cálculo $\mathrm{V}$, la condición de seguridad que debe cumplirse es la siguiente:

$$
\tau_{\text {med }}=\frac{V}{\beta \cdot A} \leq \tau_{\mathrm{a}}
$$

siendo:

$\tau_{\text {med }} \quad$ tensión media de cálculo a corte

$\mathrm{V}$ esfuerzo cortante en la base del muro

A área neta de la sección horizontal del muro

$\tau \quad$ tensión admisible a corte

${ }_{\beta}^{a}$ coeficiente de reducción de la resistencia al corte, que adopta los valores:

$$
\beta=1 \text { para } m_{b} \leq 1
$$

\section{y}

$\beta=\frac{3}{2}-\frac{3 \cdot \mathrm{e}_{\mathrm{b}}}{\mathrm{b}}$ para $-1<\mathrm{m}_{\mathrm{b}} \leq 1,3$
$* * *$ 


\section{REFERENCIAS Y COMENTARIOS}

(1) Norma Básica de la Edificación NBE FL-90: Muros resistentes de fábrica de ladrillo. MOPU. 1990

(2) EUROCODE 6. Design of Masonry Structures. CEN. Commision of the European Comunities, 1992

(3) DE ISIDRO, F., PUERTA, A., GÓMEZ, E., FOMBELLA, R., Recomendaciones para el Proyecto, Cálculo, Ejecución y Control de los Muros Resistentes de Fábrica de Ladrillo y Bloque Cerámico. HISPALYT, 1992.

(4) Esta hipótesis está del lado de la seguridad, ya que la fábrica tiene una cierta resistencia a tracción. Sin embargo, es difícil estimar dicha resistencia, ya que depende entre otras cosas del grado de calidad con que la fábrica se ejecute.

(5) Norma Básica de la Edificación NBE AE-88: Acciones en la edificación. MOPU, 1988

(6) Se recomienda considerar una disminución de la resistencia a compresión del material en elementos de área pequeña; para elementos con secciones de $0,2 \mathrm{~m}^{2}$ o menores, la resistencia debe mutiplicarse por el siguiente factor:

$$
(0,70+1,5 \mathrm{~A})
$$

donde $\mathrm{A}$ es el área del elemento resistente.

(7) La resistencia al corte de un muro depende tanto de la resistencia al corte puro del material como del grado de solicitación a compresión que tenga; es decir, un aumento de carga vertical mejora el comportamiento del material, eleva el valor de la resistencia al corte, y por lo tanto el de la tensión de comparación correspondiente.

En cualquier caso, para ladrillos con resistencias a compresión de más de $150 \mathrm{kp} / \mathrm{cm}^{2}$ el valor de $\mathrm{f}_{\mathrm{vk} 0}$ debe limitarse a un valor máximo de $3,0 \mathrm{kp} / \mathrm{cm}^{2}$ si se utilizan morteros con mezclas ricas en cemento (M80, M160), y a $1,5 \mathrm{kp} / \mathrm{cm}^{2}$ si las mezclas son pobres (M25, M40). También debe limitarse el valor de $f_{\mathrm{vk}}$ a un valor máximo de 15 $\mathrm{kp} / \mathrm{cm}^{2}$. Se recomienda tomar los valores propuestos para otros tipos de materiales en "Recomendaciones para el Proyecto, Cálculo, Ejecución y Control de los Muros Resistentes de Fábrica de Ladrillo y Bloque Cerámico". HISPALYT, 1992.

(8) En la norma española NBE FL-90 se indican diversos criterios a seguir para obtener el valor de $t_{v}$ en varios tipos de secciones complejas.

(9) Este factor es de difícil determinación debido a la propia complejidad del fenómeno de flexopandeo de un muro. Sus valores varían de unas normativas a otras, dependiendo de la experiencia que se tiene en distintos países. Se recomienda tomar los valores propuestos en "Recomendaciones para el Proyecto, Cálculo, Ejecución y Control de los Muros Resistentes de Fábrica de Ladrillo y Bloque Cerámico". HISPALYT, 1992.

(10) La rigidez de los nudos crece a medida que éstos soportan más carga. Esta circunstancia aumenta la complejidad del problema, ya que el grado de empotramiento es difícilmente evaluable. Algunas normativas europeas consideran un hipótesis simplificativa para edificios de pocas alturas, que considera los nudos muro-forjado articulados, admitiendo que se produce una plastificación. A su vez proponen valores para los coeficientes de reducción por esbeltez $y$ excentricidad que tient: en cuenta dicha hipótesis.

(11) Es aconsejable que el valor de " $r$ " no sea mayor que media asta para no producir innecesariamente grandes excentricidades. De igual forma conviene centrar los muros superiores sobre los inferiores en tramos intermedios, de manera que $r_{1}=r_{2}$

(12) El valor del coeficiente de excentricidad de cargas se limita especialmente al valor 1,30 para evitar que el muro tenga una gran parte de su sección fisurada. Debe tenerse en cuenta que un muro de arriostramiento está sometido fundamentalmente a esfuerzos de corte. Con un valor bajo de $m_{b}$ se asegura que existe una gran porción de sección no fisurada que puede resistir dichos esfuerzos.

También es recomendable el empleo de ladrillos con porcentajes de huecos bajos, con el fin de evitar el aplastamiento a compresión horizontal de los bloques en las zonas más solicitadas.

(13) Los valores de $\mathrm{m}$ inferiores a 0,30 deben considerarse como poco significativos, debiéndose tener en cuenta una cierta cautela en su empleo. En muros que tengan una función estática importante es conveniente que el coeficiente $\mathrm{m}_{\mathbf{1}}$ no sea superior a 1,8 .

NOTA: Se ha desarrollado una aplicación informática en entorno Windows para ordenadores personales basada en este método de cálculo, que permite el análisis de la sensibilidad de cada variable cómoda e interactivamente facilitando un diseño óptimo. Dicha aplicación ha sido desarrollada por Félix Hernando Mansilla y auspiciada por HISPALYT. Para mayor información, se recomienda consultar la siguiente referencia:

HERNANDO MANSILLA, F., DE ISIDRO GORDEJUELA, F., Propuesta de una metodología de cálculo para muros estructurales de fábrica de ladrillo y bloque cerámico. Nueva arquitectura con arcilla cocida,1(1995),72-78.Faenza Editrice Ibérica, HISPALYT 